\title{
Atrial fibrillation: an inherited cardiovascular disease-a commentary on genetics of atrial fibrillation: from families to genomes
}

\author{
Hayato Tada, Masa-aki Kawashiri, Masakazu Yamagishi and Kenshi Hayashi
}

Journal of Human Genetics (2016) 61, 3-4; doi:10.1038/jhg.2015.63; published online 11 June 2015

\section{ATRIAL FIBRILLATION AS AN INHERITED CARDIOVASCULAR DISEASE}

The fraction of the risk for atrial fibrilla1 tion (AF) attributable to established factors (hypertension, smoking, obesity, diabetes mellitus, age, male sex and heart disease) is roughly $50 \%$ with hypertension being the most prominent modifiable risk factor. ${ }^{1}$ On the other hand, it has been shown that at least $5 \%$ of all patients with $\mathrm{AF}$ and $15 \%$ of those with only AF but without established clinical risk factors for AF had a positive history of $\mathrm{AF}^{2}$ These facts have motivated researchers to investigate the risk of AF among those with documented familial $\mathrm{AF}$, as well as genome-wide association studies (GWASs) that have screened for common single-nucleotide polymorphisms (SNPs) associated with $\mathrm{AF}^{3}$ In this issue of the journal, Christophersen et al. ${ }^{4}$ have nicely summarized the current understanding of genetics in AF from rare variants to common variants. We acknowledge that $\mathrm{AF}$ is heritable; however, most cardiologists do not routinely collect information on family history of AF, nor do they use this information for decisionmaking in clinical practice due to the lack of evidence. Thus, future clinical trials are needed to verify the clinical utility of family history information of AF beyond established risk factors in the first place. The next step could be genotype-phenotype association studies regardless of allele frequency. Finally, one of the possible tentative goals of genetics in $\mathrm{AF}$ could be to develop a risk calculator for

H Tada, M Kawashiri, M Yamagishi and K Hayashi are at Division of Cardiovascular Medicine, Kanazawa University Graduate School of Medicine, Kanazawa, Japan

E-mail: ht240z@sa3.so-net.ne.jp
AF based on human variations. Such approaches have already been applied in AF. For example, an AF genetic risk score comprising 12 common genetic variations was as powerful as hypertension to estimate AF events. ${ }^{5}$ However, further studies with larger samples, and with multiple ethical perspectives, are needed to be accepted as a common clinical practice to investigate such genetic risk scores. Another possible goal could be to find novel molecules as therapeutic targets, as has been shown with lipids. In this regard, whole-exome sequencing (WES) has emerged as a promising tool for gene discovery in families with suspected monogenic (or polygenic) disorders, with a success rate exceeding 20\%. ${ }^{6}$ In general, hundreds or thousands of variants are found in an individual through the WES approach. ${ }^{7}$ Then, typically, the variants predicted as benign, common and unmatched assuming co-segregation should be excluded. In this process, great advances have been made in the fields of in silico variant annotation prediction and in the information of allele frequency of a certain variant based on huge collaborative efforts in exome-sequenced data sets publicly available.

\section{AF AS AN ION CHANNEL DISEASE}

Since the first gene (KCNQ1) responsible for familial AF was identified in 2003, many variants in genes encoding ion channel subunits, cardiac gap junctions and signaling molecules have been identified in monogenic AF families. These genetic variants predispose individuals to AF by enhanced or delayed atrial action potential repolarization, conduction velocity heterogeneity, cellular hyperexcitability and hormonal modulation of atrial electrophysiology. ${ }^{8}$ Once a susceptibility variant is identified, it is important to identify the mechanistic links between the variant and disease expression. The

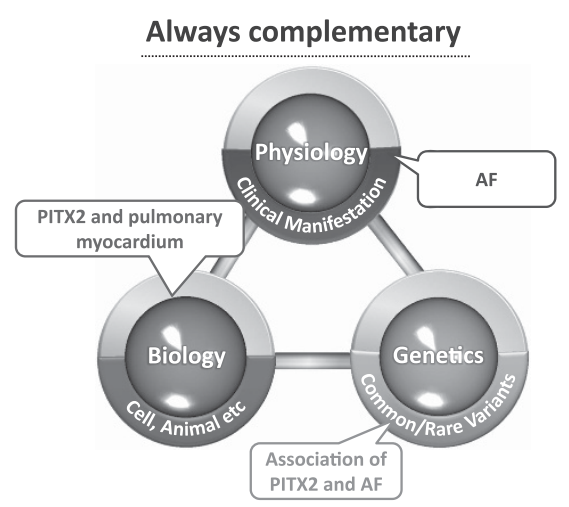

Figure 1 Schema of physiology, biology and genetics. Investigations of physiology, biology and genetics are always complementary, and have contributed to our understanding of AF. A full color version of this figure is available at the Journal of Human Genetics journal online. 
functional role of ion channel gene variants in $\mathrm{AF}$ can be usually assessed by a cellular electrophysiological study using a heterologous expression system. In addition, elucidating the molecular mechanisms of AF may allow a mechanism-based approach for treatment to be developed. For example, patients with gain-of-function ion channel variants are likely to benefit from a drug that selectively blocks mutant channel complexes. WES applied to familial AF kindreds or lone AF patients will identify many rare variants of different types of genes, and the true causal allele should be identified from the large number of insignificant alleles. To link these variants to a clinical phenotype, a family study, as well as functional evaluation, is required using a model system including zebrafish, mice or patient-specific induced pluripotent stem cells.

\section{PHYSIOLOGY, BIOLOGY AND GENETICS OF AF}

In 1998, Häissagurre et al. ${ }^{9}$ demonstrated that pulmonary veins are an important source of ectopic beats, initiating frequent paroxysms of AF. After this report, myocardial sleeve, in which paired-like homeodomain transcription factor 2 (PITX2) has a key role in its formation, has been shown to initiate AF. In 2009, a GWAS identified a strong association between SNPs in the PITX2 gene and AF. ${ }^{10}$ Each of these investigations contributed complementarily to our understanding of $\mathrm{AF}$ (Figure 1).

\section{CONCLUSION}

Given the development of novel genotyping and sequencing technologies, as well as extensive catalogs of human genetic variation together with functional analyses, cardiologists are now realizing that $\mathrm{AF}$ is highly heritable. Rigorous efforts are currently underway, and it is highly likely that these efforts could reveal the contribution of rare and common variants to the overall genetic architecture of AF in the next few years.

1 Magnani, J. W., Rienstra, M., Lin, H., Sinner, M. F., Lubitz, S. A., McManus, D. D. et al. Atrial fibrillation: current knowledge and future directions in epidemiology and genomics. Circulation 124, 1982-1993 (2011).

2 Darbar, D., Herron, K. J., Ballew, J. D., Jahangir, A., Gersh, B. J., Shen, W. K. et al. Familial atrial fibrillation is a genetically heterogeneous disorder. J. Am. Coll. Cardiol. 41, 2185-2192 (2003).
3 Ellinor, P. T., Lunetta, K. L., Albert, C. M., Glazer, N. L., Ritchie, M. D., Smith, A. V. et al. Meta-analysis identifies six new susceptibility loci for atrial fibrillation. Nat. Genet. 44, 670-675 (2012).

4 Christophersen, I. E. \& Ellinor, P. T. Genetics of atrial fibrillation: from families to genomes. J Hum Genet. 61, 61-70 (2016).

5 Tada, H., Shiffman, D., Smith, J. G., Sjögren, M., Lubitz, S. A., Ellinor, P. T. et al. Twelve-single nucleotide polymorphism genetic risk score identifies individuals at increased risk for future atrial fibrillation and stroke. Stroke 45, 2856-2862 (2014)

6 Stitziel, N. O., Peloso, G. M., Abifadel, M., Cefalù, A. B., Fouchier, S., Motazacker, M. M. et al. Exome sequencing in suspected monogenic dyslipidemias. Circ. Cardiovasc. Genet. 8, 343-350 (2015).

7 Tada, H., Kawashiri, M. A., Nohara, A., Saito, R., Tanaka, Y., Nomura, A. et al. Whole exome sequencing combined with integrated variant annotation prediction identifies asymptomatic Tangier disease with compound heterozygous mutations in ABCA1 gene. Atherosclerosis 240, 324-329 (2015).

8 Roberts, J. D. \& Gollob, M. H. A contemporary review on the genetic basis of atrial fibrillation. Methodist DeBakey Cardiovasc. J. 10, 18-24 (2014).

9 Haïssaguerre, M., Jaïs, P., Shah, D. C., Takahashi, A., Hocini, M., Quiniou, G. et al. Spontaneous initiation of atrial fibrillation by ectopic beats originating in the pulmonary veins. N. Engl. J. Med. 339, 659-666 (1998).

10 Kääb, S., Darbar, D., van Noord, C., Dupuis, J., Pfeufer, A., Newton-Cheh, C. et al. Large scale replication and meta-analysis of variants on chromosome 4q25 associated with atrial fibrillation. Eur. Heart J. 30, 813-819 (2009). 